\title{
A Panchromatic View of Relativistic Jets in Narrow-Line Seyfert 1 Galaxies
}

\author{
Filippo D'Ammando ${ }^{1,2}, *$, Monica Orienti ${ }^{2}$, Justin Finke ${ }^{3}$, Josefin Larsson ${ }^{4}$, Marcello Giroletti ${ }^{2}$ \\ and Claudia M. Raiteri ${ }^{5}$ \\ 1 Dipartimento di Fisica e Astronomia, Universitá degli Studi di Bologna, Viale Berti Pichat 6/2, \\ Bologna 40127, Italy \\ 2 INAF - Istituto di Radioastronomia, Via Gobetti 101, Bologna 40129, Italy; orienti@ira.inaf.it (M.O.); \\ giroletti@ira.inaf.it (M.G.) \\ 3 U.S. Naval Research Laboratory, Code 7653, 4555 Overlook Ave. SW, Washington, DC 20375-5352, USA; \\ justin.finke@nrl.navy.mil \\ 4 KTH, Department of Physics, and the Oskar Klein Centre, AlbaNova, SE-106 91 Stockholm, Sweden; \\ josla@kth.se \\ 5 INAF - Osservatorio Astrofisico di Torino, Via Osservatorio 20, I-10025 Pino Torinese (TO), Italy; \\ raiteri@oato.inaf.it \\ * Correspondence: dammando@ira.inaf.it
}

Academic Editors: Jose L. Gómez, Alan P. Marscher and Svetlana G. Jorstad

Received: 15 July 2016; Accepted: 18 August 2016; Published: 25 August 2016

\begin{abstract}
The discovery by the Large Area Telescope on board Fermi of variable $\gamma$-ray emission from radio-loud narrow-line Seyfert 1 (NLSy1) galaxies revealed the presence of a possible third class of Active Galactic Nuclei (AGN) with relativistic jets in addition to blazars and radio galaxies. Considering that NLSy1 are usually hosted in spiral galaxies, this finding poses intriguing questions about the nature of these objects and the formation of relativistic jets. We report on a systematic investigation of the $\gamma$-ray properties of a sample of radio-loud NLSy1, including the detection of new objects, using 7 years of Fermi-LAT data with the new Pass 8 event-level analysis. In addition we discuss the radio-to-very-high-energy properties of the $\gamma$-ray emitting NLSy1, their host galaxy, and black hole mass in the context of the blazar scenario and the unification of relativistic jets at different scales.
\end{abstract}

Keywords: galaxies: nuclei; galaxies: jets; galaxies: Seyfert; gamma-rays: general

\section{Introduction}

Since its launch on 11 June 2008, the Fermi Gamma-ray Space Telescope has opened a new era in high-energy astrophysics. The primary instrument on board Fermi, the Large Area Telescope (LAT), is a pair-conversion telescope covering the energy range from $\sim 20 \mathrm{MeV}$ up to $\sim 1 \mathrm{TeV}$ with unprecedented sensitivity and effective area [1]. One of the major scientific goals of the Fermi mission is to investigate the high-energy emission in Active Galactic Nuclei (AGN) in order to understand the mechanisms by which the particles are accelerated and the precise site of the $\gamma$-ray emission. The combination of deep and fairly uniform exposure over two orbits ( $~ 90 \mathrm{~min})$, very good angular resolution, and stable response of the LAT has allowed the production of the most sensitive, best-resolved survey of the $\gamma$-ray sky.

Before the launch of the Fermi satellite only two classes of AGN were known to generate strong relativistic jets, and therefore to emit up to the $\gamma$-ray energy range: blazars and radio galaxies, both hosted in giant elliptical galaxies [2]. The first 4 years of observation by Fermi-LAT confirmed that the extragalactic $\gamma$-ray sky is dominated by blazars, with only a few radio galaxies detected [3]. 
The discovery by Fermi-LAT of variable $\gamma$-ray emission from a few radio-loud narrow-line Seyfert 1 (NLSy1) galaxies revealed the presence of a possible third class of AGN with relativistic jets [4,5].

NLSy1 are a class of AGN identified by [6] and characterized by their optical properties: narrow permitted emission lines (FWHM $(\mathrm{H} \beta)<2000 \mathrm{~km} \cdot \mathrm{s}^{-1}$ ), flux ratio [OIII] $/ \mathrm{H} \beta<3$, and a bump due to Fe II (e.g., [7]). They also exhibit strong $X$-ray variability, steep $X$-ray spectra, substantial soft $X$-ray excess and relatively high luminosity (e.g., [8]). These characteristics seem to point to systems with smaller masses of the central black hole $\left(\mathrm{BH} ; 10^{6}-10^{8} \mathrm{M}_{\odot}\right)$ and higher accretion rates (close to or above the Eddington limit) with respect to blazars and radio galaxies. NLSy1 are generally radio-quiet (radio-loudness $R<10$ ), with only a small fraction ( $<7 \%$; [9]) classified as radio-loud. Objects with higher values of radio-loudness $(R>100)$ are even more sparse $(\sim 2.5 \%)$, while $\sim 15 \%$ of quasars are radio-loud. Considering that NLSy1 are usually hosted in spiral galaxies, their detection in $\gamma$-rays poses intriguing questions about the nature of these sources, the production of relativistic jets, and the mechanisms of high-energy emission. In this paper we discuss the radio to $\gamma$-ray properties of relativistic jets in NLSy1 galaxies.

In Section 2, we report the results of the LAT data analysis of a sample of NLSy1 over 7 years of Fermi observation, and discuss the $\gamma$-ray properties of NLSy1. In Sections 3-5 we discuss the X-ray, infrared-to-UV, and radio properties, respectively, of the $\gamma$-ray NLSy1. Results about the modelling of the broad-band spectral energy distribution (SED) of $\gamma$-ray emitting NLSy1 are presentented in Section 6. In Section 7 we discuss about BH mass measurements, host galaxies, and the jet formation for these sources. Throughout the paper the photon indices are parameterized as $d N / d E \propto E^{-\Gamma_{v}}$, where $\Gamma_{v}$ is the photon index in the different energy bands. We adopt a $\Lambda$ cold dark matter cosmology with $H_{0}=71 \mathrm{~km} \cdot \mathrm{s}^{-1} \cdot \mathrm{Mpc}^{-1}, \Omega_{\Lambda}=0.73$, and $\Omega_{\mathrm{m}}=0.27$ [10].

\section{The Fermi-LAT View of NLSy1}

Four radio-loud NLSy1 galaxies have been detected at high significance by Fermi-LAT in the first year of operation (i.e., 1H 0323+342, PMN J0948+0022, PKS 1502+036, and PKS 2004-447) and are included in the First LAT source catalog (1FGL) [11]. SBS 0846+513 was detected for the first time in $\gamma$-rays during October 2010-August 2011, when a significant increase in activity was observed by LAT [12]. Thus in the Third LAT source catalog (3FGL) five NLSy1 are reported [3]. The first $\gamma$-ray detection of FBQS J1644+2619 was reported in [13]. An unidentified $\gamma$-ray source at 0.23 deg from the radio position of FBQS J1644+2619 was included in the 3FGL. Analysing the $\gamma$-ray data collected on a longer period than that of 3FGL, i.e., during August 2008-December 2014, the localization of the $\gamma$-ray source was better constrained. Moreover, the LAT detection of FBQS J1644+2619 in November 2008-January 2009 and in July-October 2012 corresponds to periods of high optical activity, as observed in $V$-band by the Catalina Real-Time Sky Survey.

\subsection{Fermi-LAT Data Analysis}

In addition to these six NLSy1s already detected, new $\gamma$-ray emitting NLSy1 could be detected after accumulating more and more Fermi-LAT data. For this reason we analyze the first seven years of Fermi-LAT observations of a sample of 56 sources included in $[9,14-16]$ with a radio-loudness parameter higher than 10 in at least one of the previous samples. The LAT data used in this paper were collected from 5 August 2008 to 4 August 2015. During this time, the LAT instrument operated almost entirely in survey mode. The Pass 8 data [18], based on a complete and improved revision of the entire LAT event-level analysis, were used. The analysis was performed with the ScienceTools software package version v10r0p5. Only events belonging to the "Source" class (evclass $=128$, evtype $=3$ ) were used. We selected only events within a maximum zenith angle of 90 degrees to reduce contamination from the Earth limb $\gamma$-rays. The spectral analysis was performed with the instrument response functions P8R2_SOURCE_V6 using a binned maximum-likelihood method implemented in the Science tool gtlike. Isotropic ("iso_source_v06.txt") and Galactic diffuse emission ("gll_iem_v06.fit") 
components were used to model the background [17]. The normalization of both components was allowed to vary freely during the spectral fitting.

We analysed a region of interest of $30^{\circ}$ radius centred at the location of the NLSy1. We evaluated the significance of the $\gamma$-ray signal from the source by means of a maximum-likelihood test statistic (TS) defined as TS $=2 \times\left(\log L_{1}-\log L_{0}\right)$, where $L$ is the likelihood of the data given the model with $\left(L_{1}\right)$ or without $\left(L_{0}\right)$ a point source at the position of the target (e.g., [19]). The source model used in gtlike includes all the point sources from the 3FGL catalogue that fall within $40^{\circ}$ of the target. The spectra of these sources were parametrized by a power-law, a log-parabola, or a super exponential cut-off, as in the 3FGL catalogue. We also included new candidates within $10^{\circ}$ of the target from a preliminary source list using 7 years of Pass 8 data. A first maximum likelihood was performed to remove from the model the sources having TS $<25$. A second maximum likelihood was performed on the updated source model. As a result, in addition to the six NLSy1 already detected by Fermi-LAT, two new NLSy1 are detected with TS $>25$ : B3 1441+476 $(\mathrm{TS}=53)$, with a photon index $\Gamma_{\gamma}=2.65 \pm 0.15$ and a $0.1-100 \mathrm{GeV}$ flux of $(6.0 \pm 1.9) \times 10^{-9} \mathrm{ph} \cdot \mathrm{cm}^{-2} \cdot \mathrm{s}^{-1}$; NVSS J124634+023808 (TS = 79), $\Gamma_{\gamma}=2.59 \pm 0.09$ and a $0.1-100 \mathrm{GeV}$ flux of $(1.2 \pm 0.2) \times 10^{-8} \mathrm{ph} \cdot \mathrm{cm}^{-2} \cdot \mathrm{s}^{-1}$. No significant detection was obtained for the other sources, with a 2- $\sigma$ upper limit ranging between $(0.6-7.8) \times 10^{-9} \mathrm{ph} \cdot \mathrm{cm}^{-2} \cdot \mathrm{s}^{-1}$ (assuming $\Gamma_{\gamma}=2.5$ ). More details will be reported in D'Ammando, Orienti et al. (in prep.).

\section{2. $\gamma$-ray Properties}

The eight NLSy1 detected by Fermi-LAT at high significance up to now span a redshift range between 0.061 and 0.705 . The average apparent $\gamma$-ray isotropic luminosity of these sources in the 0.1-100 GeV energy band is between $10^{44}$ and $10^{47} \mathrm{erg} \cdot \mathrm{s}^{-1}$, a range of values typical of blazars. This may be an indication of a small viewing angle with respect to the jet axis, and therefore a high beaming factor for the $\gamma$-ray emission, similarly to blazars. In the same way, the average photon index $\Gamma_{\gamma}$ ranges between 2.2 and 2.8, a range of values usually observed for blazars [20]. In Figure 1 (left panel), we compare the $\gamma$-ray photon index and luminosity obtained over 7 years for the eight NLSy1 with those obtained over 4 years for the AGN included in the Third LAT Catalog of AGN (3LAC) [20]. It is clear that the NLSy1 lie in the region occupied by flat spectrum radio quasars (FSRQ), with the exception of $1 \mathrm{H} 0323+342$ and FBQS J1644+2619. However, these two sources have the lowest redshift among the eight NLSy1, and this might be the reason why their $\gamma$-ray luminosity is lower than the other NLSy1 and FSRQ.

Moreover, several strong $\gamma$-ray flares were observed from SBS 0846+513, PMN J0948+0022, PKS 1502+036, and $1 \mathrm{H} 0323+342$, reaching a peak apparent isotropic $\gamma$-ray luminosity of $\sim 10^{48} \mathrm{erg} \cdot \mathrm{s}^{-1}$ [21-25], comparable to that of bright FSRQ [20]. Variability was observed during all $\gamma$-ray flares on a daily time-scale; in case of PKS 1502+036 sub-daily variability was observed [25]. Moreover, SBS 0846+513 and PMN J0948+0022 showed a $\gamma$-ray flaring activity combined with a moderate spectral evolution, a behaviour that was already observed in bright FSRQ and low-synchrotron-peaked BL Lacs [26]. To summarize, luminosity, variability and spectral properties of these NLSy1 in $\gamma$-rays indicate a blazar-like behaviour.

NLSy1 are included neither in the First Fermi LAT Catalog of sources above $10 \mathrm{GeV}$ (1FHL; [27]) nor in the Second Catalog of hard Fermi-LAT Sources above $50 \mathrm{GeV}$ (2FHL; [28]). At Very High Energy (VHE; E > $100 \mathrm{GeV}$ ), VERITAS observations of PMN J0948+0022 were carried out a few days after the $\gamma$-ray flare observed by Fermi-LAT on 1 January 2013 (Figure 1, right panel). These observations resulted in an upper limit of $\mathrm{F}_{>0.2 \mathrm{TeV}}<4 \times 10^{-12} \mathrm{ph} \cdot \mathrm{cm}^{-2} \cdot \mathrm{s}^{-1}$ [23]. The lack of detection at VHE could be due to different reasons: (1) The distance of the source $(z=0.5846)$ is relatively large and most of the $\mathrm{GeV} / \mathrm{TeV}$ emission may be absorbed due to pair production from $\gamma$-ray photons of the source and the infrared photons from the extragalactic background light; (2) The VERITAS observations were carried out a few days after the peak of the $\gamma$-ray activity, thus covering only the last part of the $\mathrm{MeV} / \mathrm{GeV}$ flare; (3) Considering the similarities with FSRQ, a broad line region (BLR) should be 
present in these NLSy1. The presence of a BLR could produce a spectral break due to pair production, suppressing the flux beyond a few $\mathrm{GeV}$ and preventing a VHE detection. Future observations with the Cherenkov Telescope Array (CTA) will constrain the level of $\gamma$-ray emission at $100 \mathrm{GeV}$ or below.
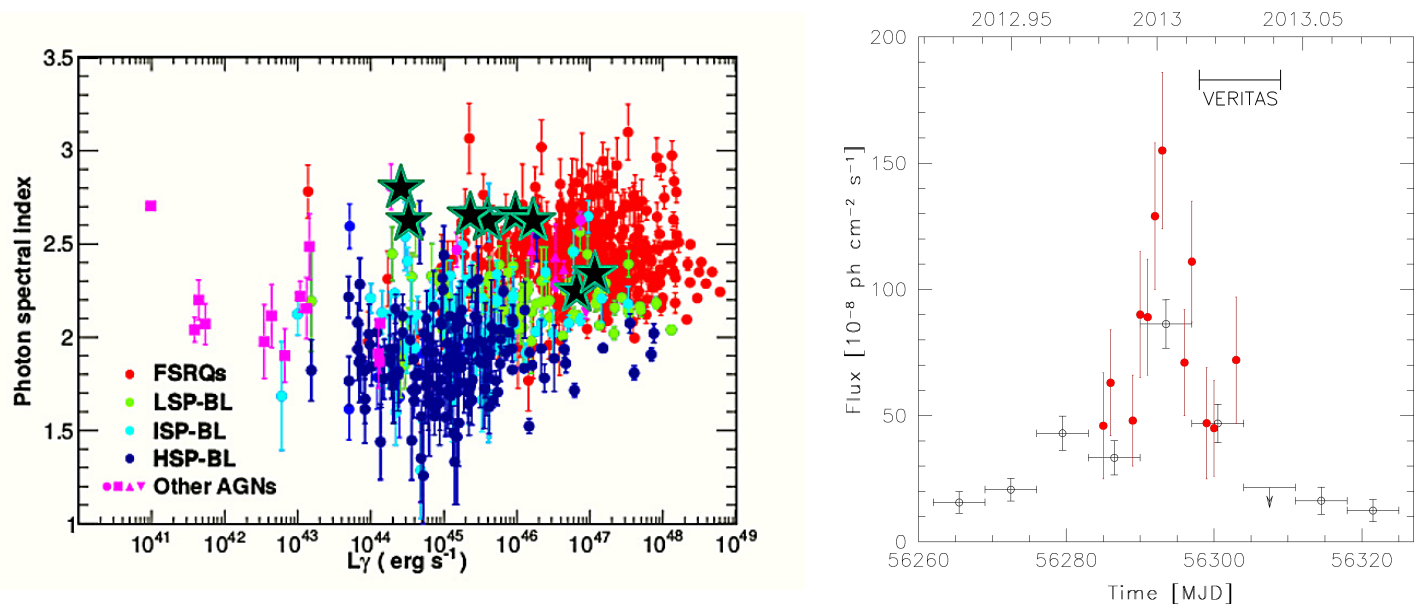

Figure 1. (Left panel): Photon index versus $\gamma$-ray luminosity of different types of Active Galactic Nuclei (AGN) included in the 3LAC. Black stars represent the values obtained over 7 years for narrow-line Seyfert 1 (NLSy1). Adapted from [20]; (Right panel): LAT light curve of PMN J0948+0022 in the 0.1-100 GeV energy range during 1 December 2012-31 January 2013 with 7-day time bins. Arrow refers to $2-\sigma$ upper limit. Upper limits are computed when $T S<10$. Red solid circles represent daily fluxes reported for the periods of high activity. The horizontal line indicates the period of the VERITAS observation. Adapted from [13].

\section{X-ray Properties}

The X-ray spectra of NLSy1 are usually characterized by a soft photon index $\left(\Gamma_{X}>2,[8]\right)$. On the contrary, a relatively hard X-ray spectrum was detected in the Swift/XRT observations of SBS 0846+513 [12,21], PMN J0948+0022 [22,23,29], 1H 0323+342 [24], PKS 1502+036 [25,30], and PKS 2004-447 [31,32]. This suggests a significant contribution of inverse Compton radiation from a relativistic jet in X-rays, similar to what is found for FSRQ (e.g., [33]).

The high quality of the XMM-Newton observation of PMN J0948+0022 performed in 28-29 May 2011 allowed us to study in detail its X-ray spectrum, as reported in [29]. The spectral fitting of the XMM-Newton data shows that emission from the jet most likely dominates the spectrum above $\sim 2 \mathrm{keV}$, while a soft $\mathrm{X}$-ray excess is evident below $\sim 2 \mathrm{keV}$. The origin of the soft $\mathrm{X}$-ray excess is still an open issue [34]. This component is a typical feature in the X-ray spectra of radio-quiet NLSy1, but it is quite unusual in jet-dominated AGN, with some exceptions (e.g., PKS 1510-089; [35]). In the case of PMN J0948+0022, the statistics did not allow us to distinguish between different models for the soft X-ray emission. Models where the soft emission is partly produced by blurred reflection, or Comptonisation of the thermal disc emission, or simply a steep power-law, all provide good fits to the data. A multicolor thermal disc emission also gives a comparable fit, but the temperature is too high $(\mathrm{kT}=0.18 \mathrm{keV})$ and is incompatible with a standard Shakura \& Sunyaev accretion disc [29]. A clear soft X-ray emission component is observed also in the XMM-Newton observation of $1 \mathrm{H} 0323+342$ collected in 23-24 August 2015 (D'Ammando et al., in prep.).

An indication of a weak soft excess was observed in the XMM-Newton spectrum of PKS 2004-447 in 2004 [36]. On the contrary, the X-ray spectra of PKS 2004-447 collected in May and October 2012 are well reproduced by a single power-law with a hard photon index $\left(\Gamma_{X} \sim 1.7\right)$ and no significant soft X-ray excess [31,32]. The XMM-Newton spectrum of PKS 1502+036 collected in 2012 was quite well fit by a simple power-law model, although some residuals are observed at low and high energies. These residuals might be a hint of the presence of the soft X-ray excess and the Iron line, respectively. 
A better fit was obtained by using a broken power-law model, suggesting the presence of two emission components in X-rays, but the uncertainties related to the spectral parameters are quite large [25].

Only one source, $1 \mathrm{H} 0323+342$, among the NLSy1 detected by Fermi-LAT was detected in hard X-rays $(E>10 \mathrm{keV})$. This source was included in the 70-month Swift-BAT catalogue, with a photon index of $1.73 \pm 0.25$ [37]. $1 \mathrm{H} 0323+342$ was included also in the Fourth IBIS/ISGRI Soft Gamma-ray Survey Catalog [38].

\section{Infrared-to-UV Properties}

Thanks to the all sky survey carried out by the Wide-field Infrared Survey (WISE) it was possible to study from a statistical point of view the infrared properties of blazars, dominated by the jet non-thermal emission, in comparison with the other extragalactic sources that are dominated by thermal emission (e.g., [39]). A dedicated study of the infrared colours of NLSy1 indicates that sources detected by LAT show infrared properties similar to FSRQ, with a dominance of the jet emission component in the infrared band [40]. By investigating WISE data, [41] found infrared intraday variability in SBS 0846+513 and PMN J0948+0022, with an amplitude of $\sim 0.1-0.2$ mag. A similar variability amplitude was observed for PKS 1502+036 on a monthly time-scale.

Optical intraday variability has been reported for PMN J0948+0022 [42,43], SBS $0846+513$ [44], and $1 \mathrm{H} 0323+342$ [24]. In the case of PMN J0948+0022 and SBS 0846+513 the intraday variability is sometimes associated with a significant increase of the optical polarisation percentage, indicating the relativistic jet as the main origin for the optical emission in these objects. The optical and UV part of the spectrum of FSRQ is usually dominated by the thermal emission of the accretion disc, while in BL Lac objects the disc is radiatively inefficient, with the disc emission overwhelmed by the non-thermal jet emission (e.g., [45]). The accretion disc emission is visible in the low activity state of the SED of PMN J0948+0022 [23], 1H 0323+342 [5] and PKS 1502+036 [25]. On the contrary, no significant evidence of thermal emission from the accretion disc has been observed in SBS $0846+513$ [21] and PKS 2004-447 [31].

\section{Radio Properties}

On pc scale a core-jet structure was observed for SBS 0846+513 [12], PKS 2004-447 [31], $1 \mathrm{H} \mathrm{0323+342} \mathrm{[46],} \mathrm{PKS} \mathrm{1502+036} \mathrm{[30],} \mathrm{and} \mathrm{PMN} \mathrm{J0948+0022} \mathrm{[29,47].} \mathrm{The} \mathrm{analysis} \mathrm{of} \mathrm{the} \mathrm{6-epoch}$ data set of SBS $0846+513$ collected by the MOJAVE programme during 2011-2013 indicates that a superluminal jet component is moving away from the core with an apparent angular velocity of $(0.27 \pm 0.02)$ mas $\cdot$ year $^{-1}$, corresponding to $(9.3 \pm 0.6) c$ [21]. This apparent superluminal velocity indicates the presence of boosting effects for the jet of SBS 0846+513. Apparent superluminal velocity of a jet component was reported also for PMN J0948+0022 and $1 \mathrm{H} \mathrm{0323+342} \mathrm{[48].} \mathrm{On} \mathrm{the} \mathrm{contrary,}$ VLBA observations did not detect apparent superluminal motion at $15 \mathrm{GHz}$ for PKS $1502+036$ during 2002-2012, although the radio spectral variability, the one-sided jet-like structure, the observed $\gamma$-ray luminosity and the Doppler factor estimated by SED modelling seem to require the presence of boosting effects in a relativistic jet [21]. A sub-luminal component was reported also in [48]. This result resembles the 'Doppler factor crisis' observed in bright TeV BL Lacs (e.g., [49]).

Strong radio variability was observed at $15 \mathrm{GHz}$ during the monitoring of the OVRO 40-m telescope of PMN J0948+0022 [23,29], PKS 1502+036 [30], SBS 0846+513 [12,21], and 1H 0323+342 [24]. An inferred variability brightness temperature of $2.5 \times 10^{13} \mathrm{~K}, 1.1 \times 10^{14} \mathrm{~K}$, and $3.4 \times 10^{11} \mathrm{~K}$ was obtained for PKS 1502+036, SBS 0846+513, and PMN J0948+0022, respectively. These values are larger than the brightness temperature derived for the Compton catastrophe [50], suggesting that the radio emission of the jet is Doppler boosted. On the other hand, a high variability brightness temperature of $10^{13} \mathrm{~K}$, comparable to that of the $\gamma$-ray NLSy1, was observed for TXS $1546+353$. However, no $\gamma$-ray emission has been detected from this source, so far [31]. Moreover, intensive monitoring of the $\gamma$-ray NLSy1 from 2.6 GHz to $142 \mathrm{GHz}$ with the Effelsberg 100-m and IRAM 30-m telescopes showed, in 
addition to an intensive variability, spectral evolution across the different bands following evolutionary paths explained by travelling shocks, typical characteristics seen in blazars [51].

On kpc scale a two-sided radio structure was detected for PMN J0948+0022, 1H 0323+342, and FBQS J1644+2619 [52], with a core-dominated structure as observed in blazars.

\section{SED Modelling of $\gamma$-ray Emitting NLSy1}

The first SED collected for the NLSy1 detected in the first year of Fermi operation showed clear similarities with blazars: a double-humped shape with a first peak in the IR/optical band due to synchrotron emission, a second peak in the $\mathrm{MeV} / \mathrm{GeV}$ band likely due to inverse Compton emission. The physical parameters of the jet of these NLSy1 are blazar-like, and the jet power is in the average range of blazars [5]. We compared the SED of SBS 0846+513 during the flaring state in May 2012 with that of a quiescent state (Figure 2, left panel). The SED of the two different activity states, modelled by an external Compton (EC) component of seed photons from a dust torus, could be fitted by changing the electron distribution parameters as well as the magnetic field [21]. A significant shift of the synchrotron peak to higher frequencies was observed during the May 2012 flaring episode, similar to FSRQ (e.g., PKS 1510-089; [33]).
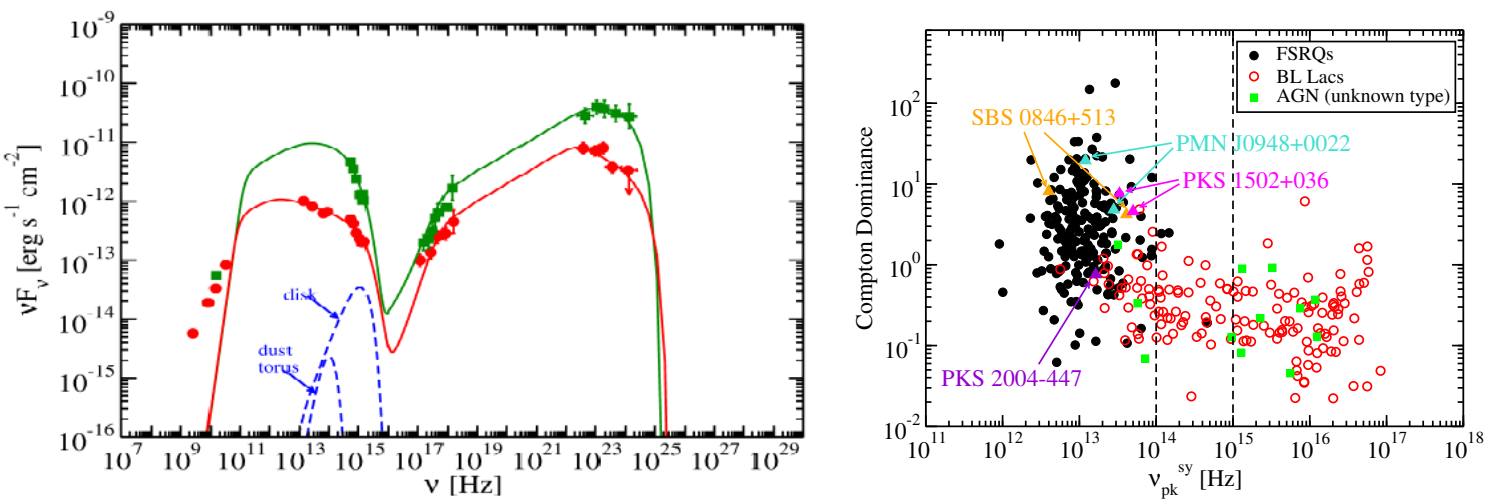

Figure 2. (Left panel): Spectral energy distribution (SED) and model fit (solid curve) of SBS $0846+513$ in flaring activity (squares) and low activity (circles) with the disk and dust torus emission components shown as dashed curves. Adapted from [21]; (Right panel): the Compton dominance versus peak synchrotron frequency for sources from the Second LAT AGN catalog (2LAC) [53], along with two activity states of SBS 0846+513 [21], PMN J0948+0022 [23], PKS $1502+036$ [25], and an average state of PKS 2004-447 [31]. Dashed lines indicate the boundary between high-synchrotron-peak, intermediate-synchrotron-peaked, and low-synchrotron-peaked blazars. Adapted from [54], reproduced by permission of the AAS.

For PMN J0948+0022 we compared the broad-band SED of the 2013 flaring activity state with that from an intermediate activity state observed in 2011. Contrary to what was observed for some FSRQ (e.g., PKS 0537-441; [55]) the SED of the two activity states, modelled as synchrotron emission and EC scattering of seed photons from a dust torus, could not be modelled by changing only the electron distribution parameters. A higher magnetic field is needed for the high activity state, consistent with the modelling of different activity states of PKS 0208-512 [56]. We also modelled the 2013 flaring state of PMN J0948+0022 assuming Compton-scattering of BLR line radiation. The model reproduces the data as well as the scattering of the IR torus photons, but requires magnetic fields which are far from equipartition between the energy densities of particles and magnetic field. In the case of PKS 2004-447, the inverse Compton component is modelled with an EC scattering of dust torus seed photons, as for the SED of SBS 0846+513 and PMN J0948+0022 [31]. For PKS 1502+036 the SED of an average activity state was compared with that of the 2015 flaring state. The two SED could be fitted with the high-energy bump modelled as an EC component with seed photons from a dust torus, 
and by changing the electron distribution parameters as well as the magnetic field. The fit of the disc emission during the average state constrains the $\mathrm{BH}$ mass to values lower than $10^{8} \mathrm{M}_{\odot}$ [25].

Following the 'classical' blazar sequence [57], [54] found a clear correlation between Compton dominance and the rest-frame peak synchrotron frequency $\left(v_{p k}^{s y}\right)$ in blazars, related to the contribution of the EC component to the high-energy bump. In Figure 2 (right panel) the Compton dominance versus $v_{p k}^{s y}$ obtained for the sources in the 2LAC is compared to the values obtained for $5 \gamma$-ray emitting NLSy1 in different activity states. NLSy1 lie in the region occupied by FSRQ, confirming that the EC is the dominant mechanism for producing the $\gamma$-ray emission in these sources, and therefore the similarity between FSRQ and these NLSy1.

\section{Black Hole Mass, Host Galaxy, and Jet Formation}

The mechanisms for producing a relativistic jet are still unclear. In particular, the physical parameters that drive the jet formation are still under debate. One of the key parameters should be the BH mass, with only large masses allowing an efficient jet formation (e.g., [58]). The most powerful jets are found in luminous elliptical galaxies with very massive BH. This was interpreted as an indirect evidence that a high spin is required for the jet production, since at least one major merger seems to be necessary to spin up the SMBH [58,59] suggested that a $\mathrm{BH}$ mass higher than $10^{8} \mathrm{M}_{\odot}$ is necessary for producing a radio-loud AGN and that the merger history together with the subsequent galaxy morphology plays a fundamental role.

The detection of variable $\gamma$-ray emission from a few NLSy1 galaxies confirmed the presence of a relativistic jet in these objects. The discovery of relativistic jets in a class of AGN usually hosted in spiral galaxies (e.g., [60]), with a $\mathrm{BH}$ mass ranging between $10^{6}$ and $10^{8} \mathrm{M}_{\odot}$, was a great surprise. This discovery challenges the current knowledge on how the structures are generated and developed (e.g., [61,62]). However, the estimated masses of the NLSy1 have large uncertainties. It was suggested that the BH masses of NLSy1 are underestimated due either to the effect of radiation pressure [63] or to projection effects [64]. Higher BH masses than those derived by the virial method (e.g., [14]) are in agreement with the values estimated by modelling the optical/UV data with a Shakura and Sunyaev disc spectrum (i.e., $10^{8}-10^{9} \mathrm{M}_{\odot}$; [65]). This may solve the problem of the minimum $\mathrm{BH}$ mass predicted in different scenarios of relativistic jet formation, but introduces a possible new issue. Spiral galaxies are formed by minor mergers, with $\mathrm{BH}$ mass typically ranging between $10^{6}$ and $10^{8} \mathrm{M} \odot$ (e.g., [66]). If the BH mass for radio-loud NLSy1 is on the larger side of the estimated values, how is it possible to reconcile such a large $\mathrm{BH}$ mass with a spiral galaxy not subject to major mergers?

Among the radio-loud NLSy 1 detected in $\gamma$-rays up to now, only for the closest one, $1 \mathrm{H} 0323+342$ $(z=0.061)$, the host galaxy structure was detected. Observations with the Hubble Space Telescope and the Nordic Optical Telescope revealed a one-armed galaxy morphology or a circumnuclear ring, respectively, suggesting two possibilities: the spiral arm of the host galaxy [67] or the residual of a galaxy merger $[68,69]$. These observations, together with the lack of information about the host galaxy of the other $\gamma$-ray emitting NLSy1, leave room for the hypothesis that the NLSy1 detected in $\gamma$-rays could have peculiar host galaxies with respect to the other NLSy1 (e.g., non-spiral morphology, undergoing strong merger activity). Further high-resolution observations of the host galaxy of $\gamma$-ray emitting NLSy1 are fundamental to obtain important insights into the onset of production of relativistic jets in these sources.

Acknowledgments: The Fermi-LAT Collaboration acknowledges support for LAT development, operation and data analysis from NASA and DOE (United States), CEA/Irfu and IN2P3/CNRS (France), ASI and INFN (Italy), MEXT, KEK, and JAXA (Japan), and the K.A. Wallenberg Foundation, the Swedish Research Council and the National Space Board (Sweden). Science analysis support in the operations phase from INAF (Italy) and CNES (France) is also gratefully acknowledged.

Author Contributions: Filippo D'Ammando analyzed the X-ray and $\gamma$-ray data, and wrote the paper; Monica Orienti analyzed the radio and $\gamma$-ray data, and contributed to write the paper; Justin Finke modelled the SED; Josefin Larsson analyzed the X-ray data; Marcello Giroletti analyzed the radio data; Claudia M. Raiteri analyzed the optical and UV data. 
Conflicts of Interest: The authors declare no conflict of interest.

\section{References}

1. Atwood, W.B.; Abdo, A.A.; Ackermann, M.; Althouse, W.; Anderson, B.; Axelsson, M.; Baldini, L.; Ballet, J.; Band, D.L.; Barbiellini, G.; et al. The large area telescope on the Fermi gamma-ray space telescope mission. Astrophys. J. 2009, 697, 1071-1102.

2. Blandford, R.D.; Rees, M.J. Some comments on radiation mechanisms in Lacertids. In Proceedings of the Pittsburgh Conference on BL Lac Objects, Pittsburgh, PA, USA, 24-26 April 1978.

3. Acero, F.; Ackermann, M.; Ajello, M.; Albert, A.; Atwood, W.B.; Axelsson, M.; Baldini, L.; Ballet, J.; Barbiellini, G.; Bastieri, D.; et al. Fermi large area telescope third source catalog. Astrophys. J. Suppl. 2015, 218, 23.

4. Abdo, A.A.; Ackermann, M.; Ajello, M.; Axelsson, M.; Baldini, L.; Ballet, J.; Barbiellini, G.; Bastieri, D.; Battelino, M.; Baughman, B.M.; et al. Fermi/large area telescope discovery of gamma-ray emission from a relativistic jet in the narrow-line quasar PMN J0948 + 0022. Astrophys. J. 2009, 699, 976-984.

5. Abdo, A.A.; Ackermann, M.; Ajello, M.; Baldini, L.; Ballet, J.; Barbiellini, G.; Bastieri, D.; Bechtol, K.; Bellazzini, R.; Berenji, B.; et al. Radio-loud narrow-line Seyfert 1 as a new class of gamma-ray active galactic nuclei. Astrophys. J. 2009, 707, L142-L147.

6. Osterbrock, D.E.; Pogge, R.W. The spectra of narrow-line Seyfert 1 galaxies. Astrophys. J. 1985, 297, $166-176$.

7. Pogge, R.W. Narrow-line Seyfert 1s: 15 years later. New Astron. Rev. 2000, 44, 381-385.

8. Grupe, D.; Komossa, S.; Leighly, K.M.; Page, K.L. The Simultaneous optical-to-X-ray spectral energy distribution of soft X-ray selected active galactic nuclei observed by swift. Astrophys. J. Suppl. 2010, 187, 64-106.

9. Komossa, S.; Voges, W.; Xu, D.; Mathur, S.; Adorf, H.-M.; Lemson, G.; Duschl, W.J.; Grupe, D. Radio-loud narrow-line Type 1 quasars. Astron. J. 2006, 132, 531-545.

10. Komatsu, E.; Smith, K.M.; Dunkley, J.; Bennett, C.L.; Gold, B.; Hinshaw, G.; Jarosik, N.; Larson, D.; Nolta, M.R.; Page, L.; et al. Seven-year wilkinson microwave anisotropy probe (WMAP) observations: Cosmological interpretation. Astrophys. J. Suppl. 2011, 192, 18.

11. Abdo, A.A.; Ackermann, M.; Ajello, M.; Allafort, A.; Antolini, E.; Atwood, W.B.; Axelsson, M.; Baldini, L.; Ballet, J.; Barbiellini, G.; et al. Fermi large area telescope first source catalog. Astrophys. J. Suppl. 2010, 188,405 .

12. D'Ammando, F.; Orienti, M.; Finke, J.; Raiteri, C.M.; Angelakis, E.; Fuhrmann, L.; Giroletti, M.; Hovatta, T.; Max-Moerbeck, W.; Perkins, J.S.; et al. SBS 0846+513: A new $\gamma$-ray-emitting narrow-line Seyfert 1 galaxy. Mon. Not. R. Astron. Soc. 2012, 426, 317-329.

13. D'Ammando, F.; Orienti, M.; Larsson, J.; Giroletti, M. The first $\gamma$-ray detection of the narrow-line Seyfert 1 FBQS J1644+2619. Mon. Not. R. Astron. Soc. 2015, 452, 520-524.

14. Yuan, W.; Zhou, H.Y.; Komossa, S.; Dong, X.B.; Wang, T.G.; Lu, H.L.; Bai, J.M.A. Population of radio-loud narrow-line Seyfert 1 galaxies with blazar-like properties? Astrophys. J. 2008, 685, 801-827.

15. Foschini, L. Evidence of powerful relativistic jets in narrow-line Seyfert 1 galaxies. 2011, arXiv:1105.0772.

16. Foschini, L.; Berton, M.; Caccianiga, A.; Ciroi, S.; Cracco, V.; Peterson, B.M.; Angelakis, E.; Braito, V.; Fuhrmann, L.; Gallo, L.; et al. Properties of flat-spectrum radio-loud narrow-line Seyfert 1 galaxies. Astron. Astrophys. 2015, 575, A13.

17. Acero, F.; Ackermann, M.; Ajello, M.; Albert, A.; Baldini, L.; Ballet, J.; Barbiellini, G.; Bastieri, D.; Bellazzini, R.; Bissaldi, E.; et al. Development of the Model of Galactic Interstellar Emission for Standard Point-source Analysis of Fermi Large Area Telescope Data. Astrophys. J. Suppl. 2011, 223 26-48

18. Atwood, W.; Albert, A.; Baldini, L.; Tinivella, M.; Bregeon, J.; Pesce-Rollins, M.; Sgrò, C.; Bruel, P.; Charles, E.; Drlica-Wagner, A. Pass 8: Toward the full realization of the fermi-LAT scientific potential, In Proceedings of the 2012 Fermi Symposium, Monterey, CA, USA, 2 November 2012.

19. Mattox, J.R.; Bertsch, D.L.; Chiang, J.; Dingus, B.L.; Digel, S.W.; Esposito, J.A.; Fierro, J.M.; Hartman, R.C.; Hunter, S.D.; Kanbach, G.; et al. The likelihood analysis of EGRET data. Astrophys. J. 1996, 461, 396-407.

20. Ackermann, M.; Ajello, M.; Atwood, W.B.; Baldini, L.; Ballet, J.; Barbiellini, G.; Bastieri, D.; Becerra Gonzalez, J.; Bellazzini, R.; Bissaldi, E.; et al. The third catalog of active galactic nuclei detected by the fermi large area telescope. Astrophys. J. 2015, 810, 14. 
21. D'Ammando, F.; Orienti, M.; Finke, J.; Raiteri, C.M.; Angelakis, E.; Fuhrmann, L.; Giroletti, M.; Hovatta, T.; Karamanavis, V.; Max-Moerbeck, W.; et al. Multifrequency studies of the narrow-line Seyfert 1 galaxy SBS 0846+513. Mon. Not. R. Astron. Soc. 2013, 436, 191-201.

22. Foschini, L.; Ghisellini, G.; Kovalev, Y.Y.; Lister, M.L.; D'Ammando, F.; Thompson, D.J.; Tramacere, A.; Angelakis, E.; Donato, D.; Falcone, A.; et al. The first gamma-ray outburst of a narrow-line Seyfert 1 galaxy: The case of PMN J0948+0022 in 2010 July. Mon. Not. R. Astron. Soc. 2011, 413, 1671-1677.

23. D'Ammando, F.; Orienti, M.; Finke, J.; Raiteri, C.M.; Hovatta, T.; Larsson, J.; Max-Moerbeck, W.; Perkins, J.; Readhead, A.C.S.; Richards, J. L.; et al. The most powerful flaring activity from the NLSy1 PMN J0948+0022. Mon. Not. R. Astron. Soc. 2015, 446, 2456-2467.

24. Paliya, V.S.; Sahayanathan, S.; Parker, M.L.; Fabian, A.C.; Stalin, C.S.; Anjum, A.; Pandey, S.B. The peculiar radio-loud narrow line Seyfert 1 galaxy 1H 0323+342. Astrophys. J. 2014, 789, 143.

25. D'Ammando, F.; Orienti, M.; Finke, J.; Hovatta, T.; Giroletti, M.; Max-Moerbeck, W.; Pearson, T.J.; Readhead, A.C.S.; Reeves, R.A.; Richards, J.L. The awakening of the $\gamma$-ray narrow-Line Seyfert 1 galaxy PKS $1502+036$. Mon. Not. R. Astron. Soc. 2016, in press.

26. Abdo, A.A.; Ackermann, M.; Ajello, M.; Atwood, W.B.; Axelsson, M.; Baldini, L.; Ballet, J.; Barbiellini, G.; Bastieri, D.; Bechtol, K.; et al. Spectral properties of bright fermi-detected blazars in the gamma-ray band. Astrophys. J. 2010, 710, 1271-1285.

27. Ackermann, M.; Ajello, M.; Allafort, A.; Atwood, W.B.; Baldini, L.; Ballet, J.; Barbiellini, G.; Bastieri, D.; Bechtol, K.; Belfiore, A.; et al. The first fermi-lat catalog of sources above $10 \mathrm{GeV}$. Astrophys. J. Suppl. 2013, $209,34$.

28. Ackermann, M.; Ajello, M.; Atwood, W.B.; Baldini, L.; Ballet, J.; Barbiellini, G.; Bastieri, D.; Becerra Gonzalez, J.; Bellazzini, R.; Bissaldi, E.; et al. 2FHL: The second catalog of hard fermi-LAT sources. Astrophys. J. Suppl. Ser. 2016, 222, 5.

29. D’Ammando, F.; Larsson, J.; Orienti, M.; Raiteri, C.M.; Angelakis, E.; Carraminana, A.; Carrasco, L.; Drake, A.J.; Fuhrmann, L.; Giroletti, M.; et al. Multiwavelength observations of the $\gamma$-ray-emitting narrow-line Seyfert 1 PMN J0948+0022 in 2011. Mon. Not. R. Astron. Soc. 2014, 438, 3521-3534.

30. D'Ammando, F.; Orienti, M.; Doi, A.; Giroletti, M.; Dallacasa, D.; Hovatta, T.; Drake, A.J.; Max-Moerbeck, W.; Readhead, A.C.S.; Richards, J.L. The ordinary life of the $\gamma$-ray emitting narrow-line Seyfert 1 galaxy PKS 1502+036. Mon. Not. R. Astron. Soc. 2013, 433, 952-961.

31. Orienti, M.; D'Ammando, F.; Larsson, J.; Finke, J.; Giroletti, M.; Dallacasa, D.; Isacsson, T.; Stoby Hoglund, J. Investigating powerful jets in radio-loud narrow-line Seyfert 1s. Mon. Not. R. Astron. Soc. 2015, 453, 4037-4050.

32. Kreikenbohm, A.; Schulz, R.; Kadler, M.; Wilms, J.; Markowitz, A.; Chang, C.S.; Carpenter, B.; Elsässer, D.; Gehrels, N.; Mannheim, K.; et al. The gamma-ray emitting radio-loud narrow-line Seyfert 1 galaxy PKS 2004-447. I. The X-ray View. Astron. Astrophys. 2016, 585, 91.

33. D'Ammando, F.; Raiteri, C.M.; Villata, M.; Romano, P.; Pucella, G.; Krimm, H.A.; Covino, S.; Orienti, M.; Giovannini, G.; Vercellone, S.; et al. AGILE detection of extreme $\gamma$-ray activity from the blazar PKS 1510-089 during March 2009. Multifrequency analysis. Astron. Astrophys. 2011, 529, A145.

34. Gierlinski, M.; Done, C. Is the soft excess in active galactic nuclei real? Mon. Not. R. Astron. Soc. 2004, 349, L7-L11.

35. Kataoka, J.; Madejski, G.; Sikora, M.; Roming, P.; Chester, M.M.; Grupe, D.; Tsubuku, Y.; Sato, R.; Kawai, N.; Tosti, G.; et al. Multiwavelength Observations of the powerful gamma-ray quasar PKS 1510-089: Clues on the jet composition. Astrophys. J. 2008, 672, 787-799.

36. Gallo, L.C.; Edwards, P.G.; Ferrero, E.; Kataoka, J.; Lewis, D.R.; Ellingsen, S.P.; Misanovic, Z.; Welsh, W.F.; Whiting, M.; Boller, T.; et al. The spectral energy distribution of PKS 2004-447: A compact steep-spectrum source and possible radio-loud narrow-line Seyfert 1 galaxy. Mon. Not. R. Astron. Soc. 2006, 370, 245-254.

37. Baumgartner, W.H.; Tueller, J.; Markwardt, C.B.; Skinner, G.K.; Barthelmy, S.; Mushotzky, R.F.; Evans, P.; Gehrels, N. The 70 month swift-BAT all-sky hard X-ray survey. Astrophys. J. Suppl. 2013, 207, 19.

38. Bird, A.J.; Bazzano, A.; Bassani, L.; Capitanio, F.; Fiocchi, M.; Hill, A.B.; Malizia, A.; McBride, V.A.; Scaringi, S.; Sguera, V.; et al. The fourth IBIS/ISGRI soft gamma-ray survey catalog. Astrophys. J. Suppl. 2010, $186,1-9$.

39. D'Abrusco, R.; Massaro, F.; Ajello, M.; Grindlay, J.E.; Smith, Howard A.; Tosti, G. Infrared colors of the gamma-ray-detected blazars. Astrophys. J. 2012, 748, 68. 
40. Caccianiga, A.; Antón, S.; Ballo, L.; Foschini, L.; Maccacaro, T.; Della Ceca, R.; Severgnini, P.; Marchã, M.J.; Mateos, S.; Sani, E. WISE colours and star formation in the host galaxies of radio-loud narrow-line Seyfert 1. Mon. Not. R. Astron. Soc. 2015, 451, 1795-1805.

41. Jiang, N.; Zhou, H.-Y.; Ho, L.C.; Yuan, W.; Wang, T.-G.; Dong, X.-B.; Jiang, P.; Ji, T.; Tian, Q. Rapid infrared variability of three radio-loud narrow-line Seyfert 1 galaxies: A view from the wide-field infrared survey explorer. Astrophys. J. 2012, 759, L31.

42. Itoh, R.; Tanaka, Y.T.; Fukazawa, Y.; Kawabata, K.S.; Kawaguchi, K.; Moritani, Y.; Takaki, K.; Ueno, I.; Uemura, M.; Akitaya, H.; et al. Minute-scale rapid variability of the optical polarization in the narrow-line Seyfert 1 galaxy PMN J0948+0022. Astrophys. J. 2013, 775, L26.

43. Maune, J.D.; Miller, H.R.; Eggen, J.R. The extreme optical variability of J0948+0022. Astrophys. J. 2013, $762,124$.

44. Maune, J.D.; Eggen, J.R.; Miller, H.R.; Marshall, K.; Readhead, A.C.S.; Hovatta, T.; King, O. The extreme behavior of the radio-loud narrow-line Seyfert 1 galaxy J0849+5108. Astrophys. J. 2014, 794, 93.

45. Ghisellini, G., Tavecchio, F., Foschini, L., Ghirlanda, G. The transition between BL Lac objects and flat spectrum radio quasars. Mon. Not. R. Astron. Soc. 2011, 414, 2674-2689.

46. Wajima, K.; Fujisawa, K.; Hayashida, M.; Isobe, N.; Ishida, T.; Yonekura, Y. Short-term radio variability and parsec-scale structure in a gamma-ray narrow-line Seyfert 1 galaxy $1 \mathrm{H} 0323+342$. Astrophys. J. 2014, 781, 75.

47. Giroletti, M.; Paragi, Z.; Bignall, H.; Doi, A.; Foschini, L.; Gabányi, K.É.; Reynolds, C.; Blanchard, J.; Campbell, R.M.; Colomer, F.; et al. Global e-VLBI observations of the gamma-ray narrow line Seyfert 1 PMN J0948+0022. Astron. Astrophys. 2011, 528, L11.

48. Lister, M.L.; Aller, M.F.; Aller, H.D.; Homan, D.C.; Kellermann, K.I.; Kovalev, Y.Y.; Pushkarev, A.B.; Richards, J.L.; Ros, E.; Savolainen, T. MOJAVE: XIII. Parsec-scale AGN jet kinematics analysis based on 19 years of VLBA observations at $15 \mathrm{GHz}$. Astron. J. 2016, 152, 12.

49. Piner, B.G.; Pant, N.; Edwards, P.G. The jets of teV blazars at higher resolution: $43 \mathrm{GHz}$ and polarimetric VLBA observations from 2005 to 2009. Astrophys. J. 2010, 723, 1150.

50. Readhead, A.C.S. Equipartition brightness temperature and the inverse Compton catastrophe. Astrophys. J. 1994, 426, 51-59.

51. Angelakis, E.; Fuhrmann, L.; Marchili, N.; Foschini, L.; Myserlis, I.; Karamanavis, V.; Komossa, S.; Blinov, D.; Krichbaum, T. P.; Sievers, A.; et al. Radio jet emission from GeV-emitting narrow-line Seyfert 1 galaxies. Astron. Astrophys. 2015, 575, A55.

52. Doi, A.; Nagira, H.; Kawakatu, N.; Kino, M.; Nagai, H.; Asada, K. Kiloparsec-scale radio structures in narrow-line Seyfert 1 galaxies. Astrophys. J. 2012, 760, 41.

53. Ackermann, M.; Ajello, M.; Allafort, A.; Antolini, E.; Atwood, W.B.; Axelsson, M.; Baldini, L.; Ballet, J.; Barbiellini, G.; Bastieri, D.; et al. The second catalog of active galactic nuclei detected by the fermi large area telescope. Astrophys. J. 2011, 743, 171.

54. Finke, J. Compton dominance and the blazar sequence. Astrophys. J. 2013, 763, 134.

55. D'Ammando, F.; Antolini, E.; Tosti, G.; Finke, J.; Ciprini, S.; Larsson, S.; Ajello, M.; Covino, S.; Gasparrini, D.; Gurwell, M.; et al. Long-term monitoring of PKS 0537-441 with Fermi-LAT and multiwavelength observations. Mon. Not. R. Astron. Soc. 2013, 431, 2481-2492.

56. Chatterjee, R.; Fossati, G.; Urry, C.M.; Bailyn, C.D.; Maraschi, L.; Buxton, M.; Bonning, E.W.; Isler, J.; Coppi, P. An optical-near-infrared outburst with no accompanying $\gamma$-rays in the blazar PKS 0208-512. Astrophys. J. 2013, 763, L11.

57. Ghisellini, G.; Celotti, A.; Fossati, G.; Maraschi, L.; Comastri, A. A theoretical unifying scheme for gamma-ray bright blazars. Mon. Not. R. Astron. Soc. 1998, 301, 451-468.

58. Sikora, M., Stawarz, L., Lasota, J.-P. Radio loudness of active galactic nuclei: Observational facts and theoretical implications. Astrophys. J. 2007, 658, 815.

59. Chiaberge, M.; Marconi, A. On the origin of radio loudness in active galactic nuclei and its relationship with the properties of the central supermassive black hole. Mon. Not. R. Astron. Soc. 2011, 416, 917-926.

60. Deo, R.P., Crenshow, D.M., Kramer, S.B. The host galaxies of narrow-line Seyfert 1 galaxies: Nuclear dust morphology and starburst rings. Astron. J. 2006, 132, 321-346.

61. Böttcher, M.; Dermer, C.D. An evolutionary scenario for blazar unification. Astrophys. J. 2002, 564, 86.

62. Marscher, A. Jets in Active Galactic Nuclei. In The Jet Paradigm: From Microquasars to Quasars; Springer: Berlin, Germany, 2009; pp.173-201. 
63. Marconi, A.; Axon, D.J.; Maiolino, R.; Nagao, T.; Pastorini, G.; Pietrini, P.; Robinson, A.; Torricelli, G. The effect of radiation pressure on virial black hole mass estimates and the case of narrow-line Seyfert 1 galaxies. Astrophys. J. 2008, 678, 693.

64. Baldi, R.; Capetti, A.; Robinson, A.; Laor, A.; Behar, E. Radio-loud narrow line Seyfert 1 under a different perspective: A revised black hole mass estimate from optical spectropolarimetry. Mon. Not. R. Astron. Soc. 2016, 458, L69-L73.

65. Calderone, G.; Ghisellini, G.; Colpi, M.; Dotti, M. Black hole mass estimate for a sample of radio-loud narrow-line Seyfert 1 galaxies. Mon. Not. R. Astron. Soc. 2013, 431, 210-239.

66. Woo, J.-H.; Urry, M. Active galactic nucleus black hole masses and bolometric luminosities. Astrophys. J. 2002, 579, 530 .

67. Zhou, H.; Wang, T.; Yuan, W.; Shan, H.; Komossa, S.; Lu, H.; Liu, Y.; Xu, D.; Bai, J.M.; Jiang, D.R. A narrow-line Seyfert 1-blazar composite nucleus in 2MASX J0324+3410. Astrophys. J. 2007, 658, L13-L16.

68. Anton, S.; Browne, I.W.A.; Marcha, M.J. The colour of the narrow line Sy1-blazar $0324+3410$. Astron. Astrophys. 2008, 490, 583-587.

69. León Tavares, J.; Kotilainen, J.; Chavushyan, V.; Añorve, C.; Puerari, I.; Cruz-González, I.; Patino-Alvarez, V.; Antón, S.; Carraminana, A.; Carrasco, L.; et al. The host galaxy of the gamma-ray narrow-line Seyfert 1 galaxy 1H 0323+342. Astrophys. J. 2014, 795, 58 .

(C) 2016 by the authors; licensee MDPI, Basel, Switzerland. This article is an open access article distributed under the terms and conditions of the Creative Commons Attribution (CC-BY) license (http:/ / creativecommons.org/licenses/by/4.0/). 